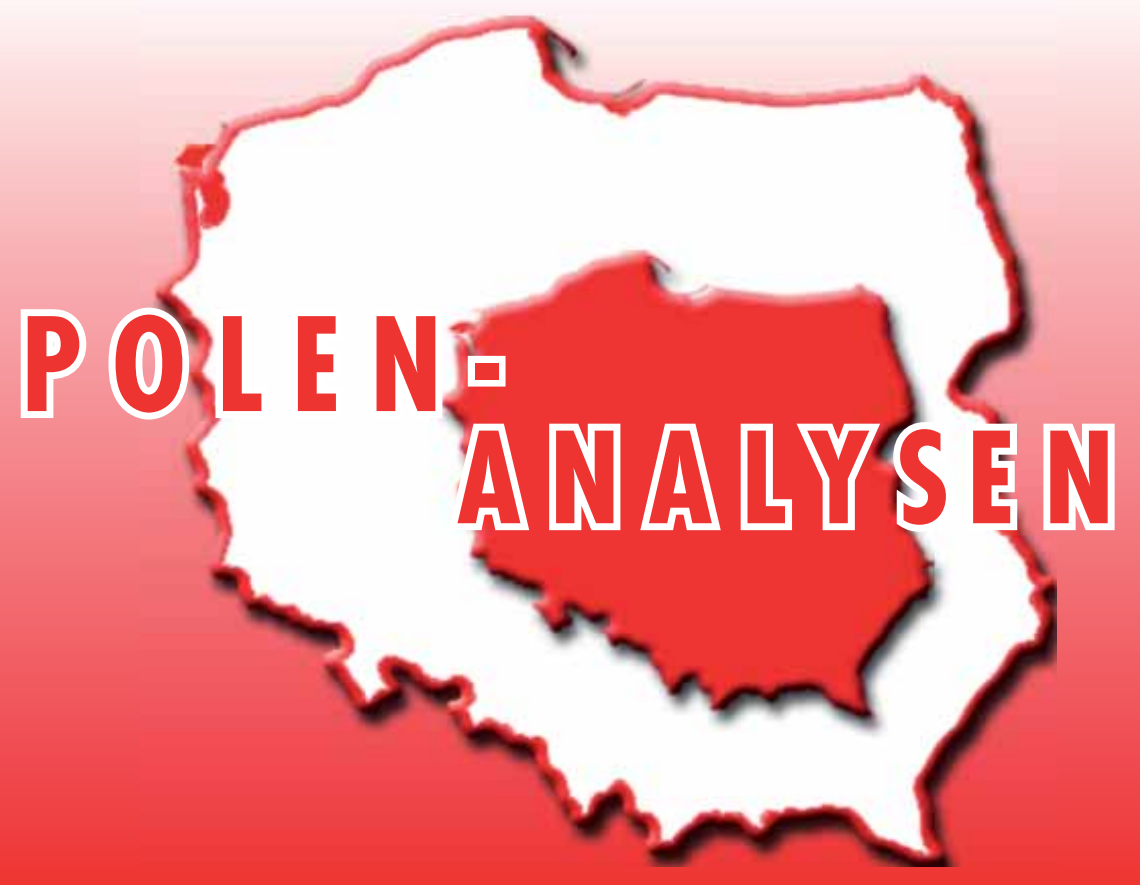

www.laender-analysen.de/polen

\title{
ARMUT IN POLEN
}

aNALYSE

Armut in Polen

Elżbieta Tarkowska, Warschau

- TABELLEN UND GRAFIKEN ZUM TEXT

Statistische Daten zur Armut in Polen

CHRONIK

Vom 19. Februar bis zum 3. März 2008

Die Herausgeber danken der BSH Bosch und Siemens Hausgeräte GmbH München und dem Stifterverband für die Deutsche Wissenschaft für ihre Unterstützung.

DEUTSCHES POLEN INSTITUT

\section{- Forschungsstelle Osteuropa}

Deutsche

Gesellschaft für Osteuropakunde e.V. 


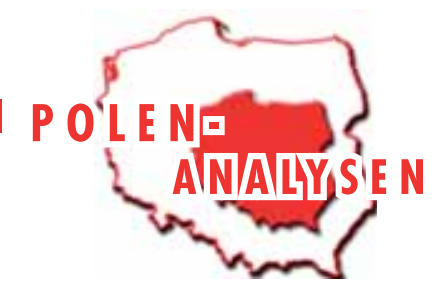

\section{Analyse}

\section{Armut in Polen}

Elżbieta Tarkowska, Warschau

\section{Zusammenfassung}

Der Text stellt die charakteristischen Formen der polnischen Armut vor. Dies sind die Armut auf dem Land, die dauerhafte Armut, die Konzentration und Regionalisierung der Armut, die Armut in zunehmend jüngeren Gesellschaftsgruppen, die spezifische Erscheinungsform der Armut bei Frauen und die Armut aufgrund von Arbeitslosigkeit und geringem Einkommen. Abschließend wird auf die Spezifik der polnischen Armut im Vergleich zu anderen Ländern hingewiesen.

A rmut in Polen ist ein komplexes, vielschichtiges und sich dynamisch veränderndes Phänomen. Diese Dynamik zeigen die Untersuchungen des Lebensstandards der Privathaushalte, die seit Jahren systematisch vom Statistischen Hauptamt (Główny Urząd Statystyczny - GUS) durchgeführt werden. Die erste Phase der Transformation wurde von einem starken Anstieg der Armut gekennzeichnet, der mit einer tiefgreifenden Restrukturierung der Wirtschaft und steigender Arbeitslosigkeit einherging. Mitte der 1990er Jahre, d.h. 1995-1996, wurde dieser negative Trend angehalten, und das Ausmaß der Armut ging zurück. Ab 1997 wuchs wiederum die Armut, die unterschiedlich definiert werden kann - und dementsprechend als extreme Armut, gesetzlich definierte Armut und relative Armut bezeichnet wird -, sukzessiv. In den Jahren 2004-2005 stabilisiert sich das Ausmaß der Armut laut dieser Definitionen, und im Jahr 2006 weisen manche Indikatoren auf eine fallende Tendenz hin. »Die Verstärkung positiver Wachstumstendenzen in den Jahren 2004-2005, die in vielen Bereichen der polnischen Wirtschaft festzustellen waren, übertrug sich nicht deutlich und unmittelbar auf die Lebensbedingungen des Durchschnittsbürgers. Darüber hinaus wurden keine wesentlichen Veränderungen hinsichtlich des Ausmaßes der nach sog. objektiven Kriterien eingeschätzten wirtschaftlichen Armut festgestellt«, schrieben die Autoren des GUS-Berichts. Veränderungen - in Form eines leichten Rückgangs (die Woiwodschaften Ermland-Masuren und Westpommern) - betreffen die subjektive Armut und die sogenannte mäßige Armut, die relativ oder gesetzlich definiert wird. Dagegen bleibt das Ausmaß der extremen Armut, die über das Existenzminimum bestimmt wird, unverändert (vgl. Tabelle auf S. 6).

Die Ergebnisse der Untersuchungen von GUS für die späteren Jahre sind noch nicht zugänglich. Allerdings zeigen andere Untersuchungen, die andere Maßstäbe für Armut anwenden, für die Jahre 2005-2007 eine Reduzierung der mäßigen Armut und gleichzeitig einen Anstieg bei den ärmsten Haushalten.
Radikale Veränderungen verzeichnet dagegen die von GUS geführte langjährige Arbeitslosenstatistik (der Anteil der registrierten Arbeitslosen an der arbeitsfähigen Zivilbevölkerung): Die Arbeitslosigkeit betrug 1990 $6,5 \%$, stieg auf ca. $20 \%$ in den Jahren 2002-2003 und fiel in den letzten Jahren auf 11-12\%; im November 2007 lag sie bei 11,2 \%. Untersuchungen zeigen aber, dass die so bedeutend kleiner gewordene Gruppe der Arbeitslosen beunruhigende Eigenschaften aufweist: Sie wird von Langzeitarbeitslosigkeit, verbunden mit Passivität, Inanspruchnahme sozialer Hilfsangebote und negativen Begleiterscheinungen, charakterisiert.

Indirekte Hinweise auf eine Verbesserung der materiellen Situation polnischer Familien können Meinungsumfragen (durchgeführt vom Zentrum zur Untersuchung der öffentlichen Meinung, Centrum Badania Opinii Społecznej - CBOS) geben, die die materielle Lage der Familien, ihre Besitzverhältnisse und finanziellen Strategien sowie die gesellschaftliche Stimmung betreffen. Sie zeigen einen wachsenden Optimismus in der Bevölkerung, was zu einem Rückgang von typischen Strategien zur Verbesserung der materiellen Situation führt, also z. B. Ausgabenbeschränkung, Aufnahme von Anleihen, Rückgriff auf finanzielle Hilfe von Verwandten, Freunden oder Institutionen.

Trotz dieser positiven Signale muss unterstrichen werden, dass immer noch ein Drittel der Gesellschaft sehr bescheiden lebt, 15-17\% in mäßiger Armut und über $10 \%$ in extremer Armut. Selbst wenn bestimmte Indikatoren auf den Rückgang der Armut hinweisen, muss doch dieser Teil der Gesellschaft im Blickfeld bleiben, d.h. die (mindestens) $10 \%$, die weiterhin mit sehr schwierigen Lebensbedingungen zu kämpfen haben. Zwar sank die Zahl der Arbeitslosen erheblich, vielleicht gibt es auch etwas weniger Arme, aber die Ausprägungen der Armut und Arbeitslosigkeit, die noch bestehen - extreme Armut und Langzeitarbeitslosigkeit - sind für die Betroffenen äußerst belastend und für die Sozialpolitik sehr schwer zu bekämpfen. 


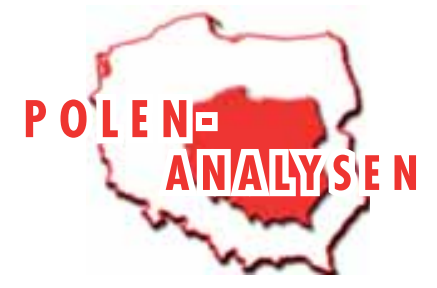

Wo auf der gesellschaftlichen Landkarte Polens ist Armut zu finden? Wer sind die polnischen Armen? Wodurch wird die polnische Armut charakterisiert? Auf der Makroebene, bezogen auf die gesamte Gesellschaft, sind die Menschen, die in Armut leben, vor allem: 1) Einwohner von Dörfern und Kleinstädten, 2) Bewohner armer Regionen sowie 3) Bewohner von Gebieten mit hoher struktureller Arbeitslosigkeit. Auf der Mikroebene des Individuums und der Familie ist Armut verbunden mit: 1) jungem Alter, 2) einem niedrigen Bildungsniveau, 3) der Familienstruktur (sie betrifft v. a. kinderreiche Familien und Alleinerziehende), 4) der Tatsache, ob das Familienoberhaupt arbeitet (Armut fördert Arbeitslosigkeit und begünstigt, dass der Haushalt mit Hilfe sog. Nicht-Erwerbs-Quellen, z. B. Wohltätigkeitsorganisationen, unterhalten wird), 5) pathologischen Zuständen wie Behinderung, chronische Krankheit, Sucht etc.

Im Weiteren werde ich folgende Eigenschaften der polnischen Armut darstellen: 1) die polnische Armut als Armut v. a. auf dem Land, 2) Armut als langfristiges, hartnäckiges, chronisches oder sogar über Generationen weitergereichtes Phänomen, 3) die Konzentration und Regionalisierung der Armut und die damit verbundenen negativen Folgen, die zum gesellschaftlichen Ausschluss führen, 4) die »Verjüngung« der polnischen Armut (Kinderarmut, Armut kinderreicher Familien, Arbeitslosigkeit der jungen Generation etc.), 5) das spezifische Phänomen der Armut bei Frauen („Feminisierung« der Armut), 6) Armut, die von Arbeitslosigkeit oder gering bezahlter Arbeit herrührt (das Phänomen der working poor).

\section{Stadt - Land}

Aus allen Untersuchungen und statistischen Analysen, die in den letzten Jahren durchgeführt wurden, geht hervor, dass die niedrigste Armutsquote in den Großstädten zu verzeichnen ist, während die meisten Armen auf dem Land und in Kleinstädten leben. Im Jahr 2005 lag die extreme Armut in den Städten bei 8,2 \%. Auf dem Land war sie über zweimal so hoch und lag bei $18,7 \%$. $12,5 \%$ der Stadtbewohner lebten in mäßiger Armut, während es auf dem Land $27 \%$ waren. Die Armut, die einerseits durch die Auflösung der Staatsgüter (Państwowe Gospodarstwo Rolne - PGR) und die damit einhergehende Arbeitslosigkeit verursacht wurde, und andererseits durch die geringe Produktivität der kleinen landwirtschaftlichen Betriebe, die vorwiegend für den eigenen Bedarf produzieren, ist eines der wichtigsten sozialen (und wirtschaftlichen ) Probleme der ländlichen Gebiete. Neben der hohen Arbeitslosigkeit kommen das niedrige Bildungsniveau der Landbevölkerung und die fehlenden Perspektiven für die junge Generation in der lokalen Gemeinschaft hinzu. In den ver- gangenen Jahren verbesserten sich die Lebensbedingungen der Bauern (z. B. stiegen ihre Einkünfte 2004 um 8,7\%), was zum einen die Einführung von Programmen der Europäischen Union bewirkte und zum anderen die Öffnung des europäischen Arbeitsmarktes. Dennoch ist die Situation der Landbewohner, die keine eigene Landwirtschaft besitzen und deren Unterhalt auch nicht durch Berufs- oder Altersrenten (sondern mit Hilfe anderer "Nicht-Erwerbs-Quellen«) bestritten wird, weiterhin sehr schwierig. Unter diesen Personen betrug die Quote extremer Armut 200445 \%. Die Armut auf dem Land ist also in sich sehr differenziert. Diese nicht nur wirtschaftlich, sondern in vielerlei Hinsicht schwierige Situation auf dem Land veranlasst manche Experten, vom Land als »dem anderen Polen« oder "Polen II $«$ zu sprechen.

\section{Armut als dauerhafter Zustand}

Die Untersuchungen, die in Polen durchgeführt wurden, insbesondere diejenigen qualitativer Art, zeigten schon in den ersten Jahren der Transformation, dass die polnische Armut - entgegen manchen Behauptungen nicht immer "oberflächlich und vorübergehend «, sondern tiefgreifend und mit einer vielschichtigen Deprivation verbunden ist und dabei lang andauernd, hartnäckig, ja sogar chronisch ist. Manchen Analysen zufolge leben $10 \%$ der Bevölkerung in dauerhafter Armut.

Einige Untersuchungen zeigen eine zunehmende Leichtigkeit, der Armut zu entkommen. Dies betrifft in erster Linie die mäßige und kurzfristige Armut. Die Stabilisierung der Quote der extremen Armut sowie bestimmte Charakteristika derer, die immer noch in Armut und Arbeitslosigkeit leben, legen aber nahe, dass das Problem der dauerhaften und dabei tiefgreifenden Armut, also derjenigen, die am schwierigsten zu bekämpfen ist, ein schwerwiegendes gesellschaftliches Problem bleibt, unabhängig von der Verbesserung der wirtschaftlichen Situation der Gesellschaft. Dies belegt auch das Problem der Obdachlosigkeit.

In polnischen Untersuchungen und Diskussionen zur dauerhaften Armut taucht als gesondertes Problem das der »Vererbung «, der Weitergabe von Armut, Arbeitslosigkeit und anderer nachteiliger Umstände von einer Generation zur nächsten, als hartnäckigstes und am schwierigsten zu bekämpfendes Problem auf. Untersuchungen zeigen unterschiedliche Versionen des schwierigen Starts ins Erwachsenenleben von jungen Menschen aus armen Familien. Diese können nicht mit der Hilfe der Eltern im Prozess des Selbständigwerdens rechnen, mehr noch, manchmal treten sie mit schwerem Ballast in die Selbständigkeit ein, nämlich mit der Notwendigkeit, die in Armut lebenden Eltern und jüngeren Geschwister zu unterstützen. Die Untersuchungen signalisieren auch das Phänomen, dass der Prozess des »Vererbens" 


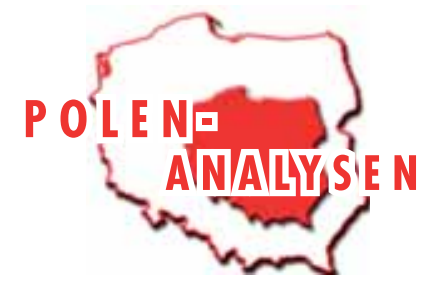

v. a. Frauen betrifft: Töchter aus armen Familien sind stärker als die Söhne gefährdet, die Armut zu erben. Als allein erziehende Mütter, ohne Qualifikationen und ohne Arbeit, sind sie auf die Wohltätigkeit sozialer Einrichtungen, karitativer Organisationen und der Familie angewiesen. Dagegen fällt es den mobileren Söhnen aus armen Familien leichter, eine physische Arbeit zu finden, die keine Qualifikationen erfordert, und selbständig zu werden. Bei vielen jungen Menschen scheinen sich die Probleme u. a. dank der Erwerbsemigration zu lösen. Untersuchungen zeigen auch die überaus wichtige Rolle von Ausbildung und Ausbildungsbemühungen, um aus dem Kreis von Armut, Arbeitslosigkeit und fehlenden Perspektiven nachhaltig auszubrechen.

\section{Regionale und lokale Konzentration der Armut}

Eine weitere wesentliche Eigenschaft der polnischen Armut ist ihre Konzentration sowohl auf der Makroebene, in regionaler Hinsicht, als auch auf der Ebene der lokalen Gesellschaften der einzelnen Städte, Stadtteile und Dörfer. Die sogenannte neue Armut tritt in Regionen auf, die bereits vor der Transformationsphase von Armut betroffen waren (das Gebiet im Nordosten und Osten Polens, d.h. die Woiwodschaften ErmlandMasuren, Heiligkreuz, Podlachien, Kujawien-Pommern, Vorkarpaten). Dabei erfährt wiederum Nordpolen (die Woiwodschaften Ermland-Masuren und Westpommern) die höchste Arbeitslosigkeit und den größten Mangel an Arbeitsplätzen, also die Gebiete der ehemaligen PGR.

Die Nivellierung der regionalen Ungleichheiten auf der Makroebene ist eine der Prioritäten der Politik der Europäischen Union, die auch in Polen umgesetzt wird. Auf die Resultate dieser Programme und Maßnahmen wird man allerdings noch warten müssen.

Neben der regionalen Differenzierung der Armut muss auch auf die Konzentration der Armut und anderer negativer Erscheinungen auf der Mikroebene der Städte, Stadtteile und Dörfer hingewiesen werden. Einerseits ist eine Ballung der armen Bevölkerung zu beobachten Enklaven von Armut in heruntergekommenen Stadtteilen mit Wohnverhältnissen, die nicht dem heutigen Standard entsprechen, - andererseits entstehen abgeriegelte reiche Wohnsiedlungen. Die Prozesse des Ausschlusses und der Isolation im städtischen Raum nehmen weiter zu.

\section{Armut bei Kindern und Jugendlichen}

Ein weiterer charakteristischer Zug der gegenwärtigen Armut in Polen ist die Verarmung und der drohende gesellschaftliche Ausschluss von Kindern, Jugendlichen und kinderreichen Familien und die angesichts der hohen Arbeitslosigkeit sehr schwierige Lage der jungen
Generation. 2005 waren es, ähnlich wie in den vorangegangenen Zeiträumen, Kinder und Jugendliche, die über $40 \%$ der in extremer Armut Lebenden ausmachten. Die Quote der extremen Armut unter Kindern bis 14 Jahre betrug ca. $19 \%$. Der UNICEF-Bericht aus dem Jahr 2007 verdeutlicht, dass polnische Kinder unter den Kindern von 24 wirtschaftlich entwickelten Ländern die ärmsten sind.

Die Wahrscheinlichkeit eines Lebens in Armut steigt mit wachsender Kinderzahl in der Familie. 2004 lebten 40,1 \% der Angehörigen kinderreicher Familien unter dem Existenzminimum, während der betreffende Indikator für die gesamte Gesellschaft 11,8 \% betrug. Das bedeutet, dass das Ausmaß der Armut in kinderreichen Familien ungefähr viermal so hoch war wie im gesellschaftlichen Durchschnitt. Hinzuzufügen wäre, dass die Quote extremer Armut dieser Familien im Jahr 2005 auf bis zu $44 \%$ gestiegen ist. Die entsprechenden Zahlen für Familien mit drei Kindern betrugen $22 \%$, für Alleinerziehende ca. $15 \%$. Demnach sind kinderreiche Familien in Polen anfälliger für Armut als Familien Alleinerziehender (Mütter), die in Westeuropa oder den USA zu den Ärmsten gehören. Es gibt allerdings auch Bereiche, in denen Kinder aus Familien jeden Typs und jeder Struktur, auch aus Familien mit wenigen Kindern, Armut und Elend erfahren.

Ein weiteres wichtiges Problem im Zusammenhang mit der Armut in immer jüngeren Bevölkerungsgruppen ist die sehr hohe Jugendarbeitslosigkeit (sie ist zweimal so hoch wie in der Gesamtbevölkerung und in bestimmten Zeitspannen erreichte sie bis zu $40 \%$ in der Altersgruppe von 15-24 Jahre) und der Mangel an Arbeitsplätzen am Wohnort bzw. in der Umgebung für die Absolventen diverser Schultypen und Ausbildungsniveaus. Die gesellschaftlichen Folgen sind das Gefühl der Perspektivlosigkeit, die Verzögerung der Selbständigkeit und die Erwerbsemigration als Bewältigungsstrategien. Diese Probleme sind derzeit Gegenstand sozialwissenschaftlicher Untersuchungen, doch das Ausmaß, die Komplexität und Dynamik dieser Erscheinungen zeigen, dass die bisherigen Erkenntnisse immer noch nicht ausreichend sind.

\section{Frauen und Armut}

Eine der charakteristischen Eigenschaften von Armut in der modernen Welt ist, dass zunehmend Frauen von ihr betroffen sind (»Feminisierung« der Armut). Nach Auffassung einiger Wissenschaftler trifft dieses Phänomen auch auf Polen zu (obgleich in dieser Frage keine Einigkeit besteht). Symptome dieses Phänomens lassen sich wahrnehmen 1) auf dem Arbeitsmarkt (höhere Arbeitslosigkeit unter Frauen, die insbesondere von Langzeitarbeitslosigkeit und den Schwierigkeiten, auf den Arbeitsmarkt zurückzukehren, betroffen sind usw.), 


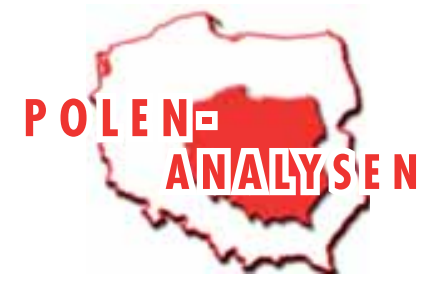

2) im Bereich des Einkommens (das Einkommen von Frauen ist durchschnittlich 20-30 \% niedriger als das der Männer), 3) im Bereich der Renten (die im Fall von Frauen durchschnittlich $20 \%$ niedriger als bei Männern sind), 4) in Form der allein erziehenden Mütter und der Haushalte, die von Frauen geführt werden, 5) auf der Mikroebene der Familie und des Haushalts (Armut bedeutet für Frauen zusätzliche Verpflichtungen, mehr Verantwortung und weniger Zeit; die Frauen sind "Armutsmanager" der Familie). In diesen Zusammenhang gehört auch die bereits genannte v. a. Frauen betreffende »Vererbung der Armut« sowie die Gefahr der Verarmung und des gesellschaftlichen Ausschlusses von zukünftigen Rentnern, insbesondere Rentnerinnen, die heute arbeitslos sind oder eine gering bezahlte Tätigkeit ausüben. Bereits in naher Zukunft werden diese nur sehr niedrige Renten erhalten.

Darüber hinaus muss unterstrichen werden, dass sowohl Frauen als auch Männer mit ihrer Gesundheit für das Leben in Armut bezahlen. Neben vielen somatischen Krankheiten (Herz- und Kreislauferkrankungen, Knochenerkrankungen, Tumore) leiden Frauen häufig an verschiedenen Neurosen und Depressionen, Männer an Alkoholismus.

\section{Armut, Arbeit und Arbeitslosigkeit}

Armut im heutigen Polen ist sehr eng mit Arbeitslosigkeit verknüpft, obgleich auch das Phänomen der working poor existiert, der armen arbeitenden Bevölkerung, die für ihre Tätigkeit nur einen sehr niedrigen Lohn erhält. Der Ausschluss vom Arbeitsmarkt sowie die gering bezahlte Arbeit sind "zwei klassische Ursachen für Armut in der Industriegesellschaft«. 2005 betrug die Quote der extremen Armut in Haushalten mit einem Arbeitslosen $27 \%$, in Haushalten ohne Arbeitslose dagegen $9 \%$. Die gering bezahlte physische Arbeit wiederum wurde von extremer Armut in $15 \%$ der Fälle begleitet, während es bei nicht-physischen Tätigkeiten $2 \%$ waren.

Als die Arbeitslosigkeit auf 11-12 \% fiel, drängten sich Phänomene wie gering bezahlte Tätigkeiten und Tätigkeiten in der Grauzone zur Schwarzarbeit in den Vordergrund. Das Ausmaß der gering bezahlten Arbeit ist in Polen sehr groß: 2005 erhielten 18,2\% der Beschäftigten $50 \%$ und weniger des durchschnittlichen Monatslohns in der nationalen Wirtschaft. Einigen Schätzungen zufolge ist die Gruppe der working poor, zusammen mit den Teilzeitbeschäftigten, die ebenfalls niedrige Löhne bekommen, in Polen sehr groß und umfasst ca. 2 Mio. Menschen.

Die gering bezahlte Arbeit und die nicht registrierte Tätigkeit in der Grauzone haben verschiedene negative Folgen. Zwar erlauben sie, in der Gegenwart über die Runden zu kommen, für die Zukunft bedeuten sie aber sehr niedrige Renten. Die so Beschäftigten werden die größte gesellschaftlich-ökonomische Gruppe, die in Armut lebt, d.h. diejenigen, die ihren Unterhalt aus sog. Nicht-Einkommens-Quellen erhalten, verstärken. 2005 lebten 29,9\% dieser Gruppe in extremer Armut.

\section{Schlussbemerkung}

Schließlich sollen die Spezifik, die Ähnlichkeiten und die Unterschiede der Armut in Polen zu anderen Ländern in Beziehung gesetzt werden. Ähnlichkeiten sind die wachsende Kluft zwischen Arm und Reich, die räumliche Trennung, das Phänomen der dauerhaften Armut in räumlich abgegrenzten Enklaven, die Konzentration der Armut und weiterer negativer Erscheinungen in bestimmten Regionen und die Verbindung zwischen Armut, Arbeitslosigkeit und niedrigem Bildungsstand.

Darüber hinaus bestehen auch Unterschiede: Armut in Polen betrifft die ländlichen Gebiete, während Armut in den westlichen Ländern mit kleinen Ausnahmen vorwiegend ein städtisches Phänomen ist. Dass die Armen in Polen jung sind und die nicht allzu schlechte Situation der Alten, der Rentner, macht die polnische Armut derjenigen in den entwickelten Ländern ähnlich. Allerdings unterscheidet sich davon die polnische Armut durch das Ausmaß der unzureichend ernährten Kinder und der Vernachlässigung ihrer Bedürfnisse. Ein weiteres gemeinsames Problem ist das Phänomen, dass Armut verstärkt Frauen betrifft, was allerdings in verschiedenen Formen auftritt. In den westlichen Ländern und den USA stellt sich diese »Feminisierung « als Armut der allein erziehenden Mütter dar, die von Sozialhilfe leben. In Polen handelt es sich dabei um die Armut von Familien mit berufstätigen Müttern und um verschiedene Formen der Ungleichheit, die geschlechtsbedingt sind und sowohl auf dem Arbeitsmarkt als auch im Privathaushalt bestehen. Der größte Unterschied ist allerdings, dass es in Polen keinen ethnischen Faktor gibt, der in den meisten Ländern den charakteristischen Zug der Armut ausmacht. Während in allen post-kommunistischen Ländern die Minderheit der Roma zu den ärmsten Teilen der Bevölkerung gehört, ist ihr Anteil in Polen nicht groß und darüber hinaus wirtschaftlich differenziert. Ein bedeutender Teil der Vertreter dieser Minderheit lebt zwar auch in Polen in Armut oder sogar im Elend, ohne Arbeit, Qualifikationen, mit niedrigem Bildungsstand und gesellschaftlich isoliert. Das Ausmaß dieser Erscheinung ist aber angesichts der zahlenmäßig kleinen Gruppe dieser Minderheit in Polen nicht groß.

Übersetzung aus dem Polnischen: Silke Plate 


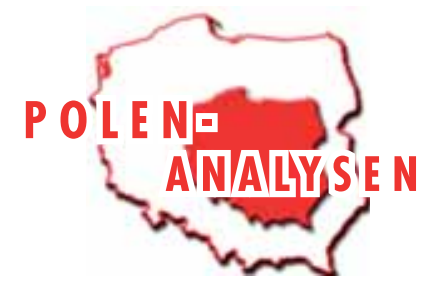

Über die Autorin

Prof. Dr. Elżbieta Tarkowska ist Soziologin und arbeitet am Institut für Philosophie und Soziologie der Polnischen Akademie der Wissenschaften (Polska Akademia Nauk - PAN) sowie an der Akademie für Sonderpädagogik in Warschau. Seit vielen Jahren forscht sie zum Thema Armut und ihrer Folgen. Weitere Spezialgebiete sind die Soziologie der Kultur, die Soziologie und Anthropologie der Zeit, Untersuchungen zum Lebensstil sowie die französische Soziologie. Sie ist Autorin zahlreicher Publikationen und Chefredakteurin der Vierteljahresschrift "Kultura i Społeczeństwo» (Kultur und Gesellschaft).

\section{Tabellen und Grafiken zum Text}

\section{Statistische Daten zur Armut in Polen}

Armut in den Jahren 1997-2006 (Betroffene in \%)

\begin{tabular}{|l|r|r|r|r|r|r|c|c|c|c|}
\hline Armutsgrenze & 1997 & $\mathbf{1 9 9 8}$ & $\mathbf{1 9 9 9}$ & $\mathbf{2 0 0 0}$ & $\mathbf{2 0 0 1}$ & $\mathbf{2 0 0 2}$ & $\mathbf{2 0 0 3}$ & $\mathbf{2 0 0 4}$ & $\mathbf{2 0 0 5}$ & $\mathbf{2 0 0 6}$ \\
\hline Existenzminimum & 5,4 & 5,6 & 6,9 & 8,1 & 9,5 & 11,1 & 10,9 & 11,8 & 12,3 & k.A. \\
$\begin{array}{l}\text { Gesetzlich definierte } \\
\text { Armut }\end{array}$ & 13,3 & 12,1 & 14,4 & 13,6 & 15,0 & 18,5 & 18,1 & 19,2 & 18,1 & 15,1 \\
Relative Armut* & 15,3 & 15,8 & 16,5 & 17,1 & 17,0 & 18,4 & 20,4 & 20,3 & 18,1 & 17,7 \\
\hline Subjektive Armut** & 30,8 & 30,8 & 34,8 & 34,4 & 32,4 & 30,4 & 28,0 & 27,3 & 22,5 & 18,3 \\
\hline
\end{tabular}

* $50 \%$ der durchschnittlichen Ausgaben eines Haushalts

** Leyden Poverty Line (LPL)

Quelle: Warunki życia ludności Polski w latach 2004-2005 [Die Lebensbedingungen der Bevölkerung in Polen in den Jahren 2004-2005], Warszawa, GUS [Główny Urzad Statystyczny, Statistisches Hauptamt], 2007, S. 71.

Wie wird sich Ihrer Meinung nach die wirtschaftliche Situation in Polen im nächsten Jahr entwickeln?

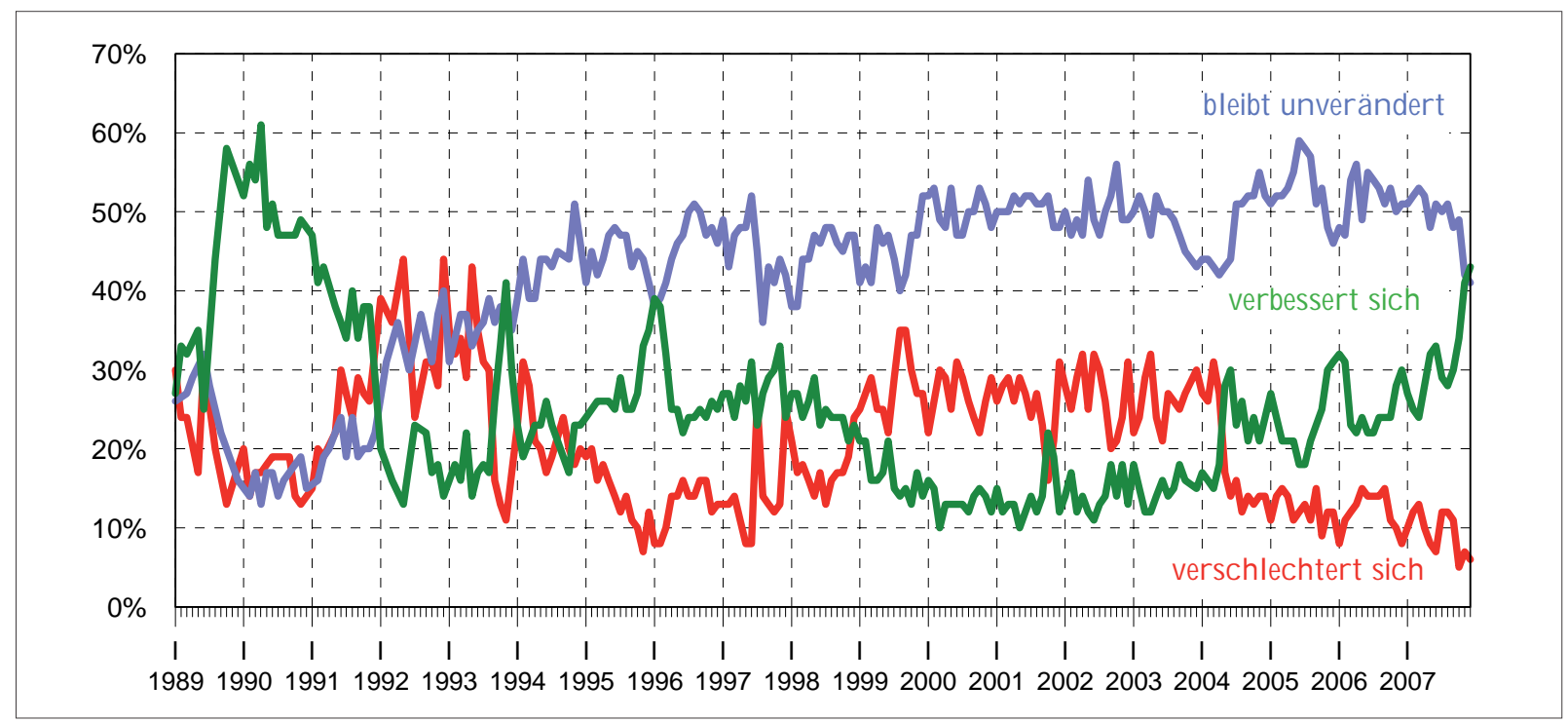

Quelle: CBOS BS/185/2007: Bardzo dobre nastroje społeczne pod koniec roku. [Sehr gute Stimmung am Ende des Jahres.], Warszawa, 12/2007, www.cbos.pl 


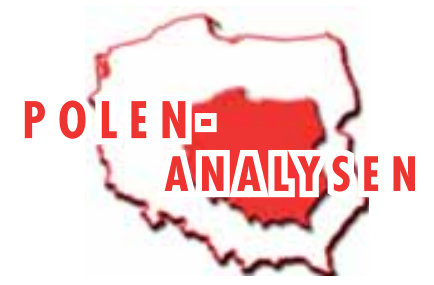

Die Arbeitslosenquote nach Woiwodschaften (Stand: Ende Dezember 2007)

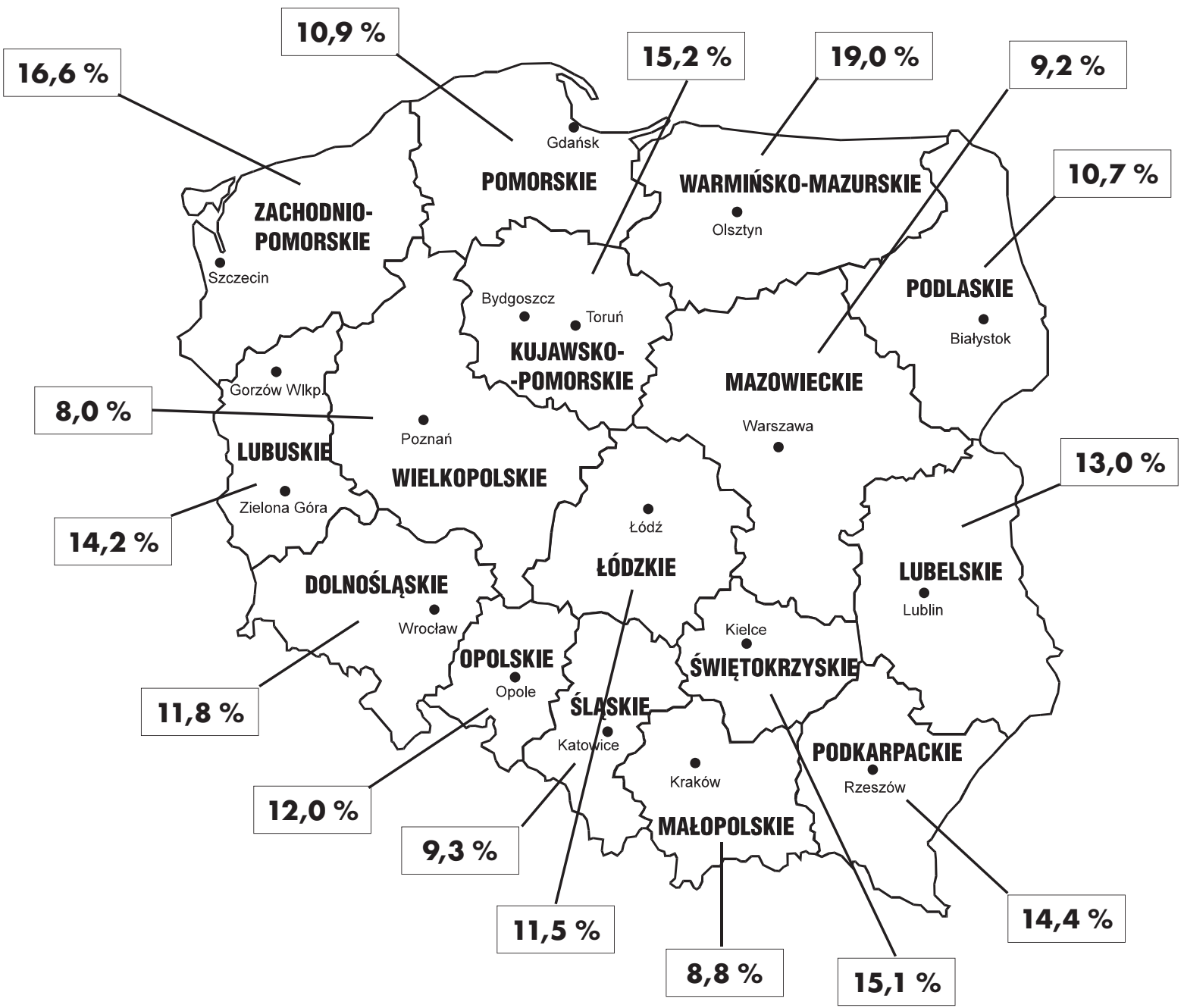

Polen insgesamt

$11,4 \%$

Białystok

$8,1 \%$

Olsztyn / Allenstein

$4,5 \%$

Bydgoszcz / Bromberg

$6,3 \%$

Opole / Oppeln

$5,4 \%$

Gdańsk / Danzig

$3,7 \%$

Poznań / Posen

$2,9 \%$

Gorzów Wielkopolski /

$6,1 \%$

Rzeszów

$7,4 \%$

Landsberg an der Warthe

Szczecin / Stettin

$6,5 \%$

Katowice / Kattowitz

$3,4 \%$

Kielce

$10,2 \%$

Kraków / Krakau

$3,8 \%$

Toruń / Thorn

$6,4 \%$

Warszawa / Warschau

$3,0 \%$

Łódź / Lodsch

$8,7 \%$

$8,3 \%$

Wrocław / Breslau

$4,7 \%$

Zielona Góra / Grünberg 7,2\%

Lublin

Quelle für Daten: Gtówny Urząd Statystyczny [Statistisches Hauptamt]: Liczba bezrobotnych oraz stopa bezrobocia wedtug województw, podregionów i powiatów. Stan w końcu grudnia 2007r. [Die Zahl der Arbeitslosen sowie die Arbeitslosenquote nach Woiwodschaften, Regionen und Kreisen. Stand: Ende Dezember 2007.], w w w.sta t.go v.pl

Quelle für Karte: CBOS, BS/13/99, Warszawa, 01/1999, http:/ / w ww.cbos.pl 


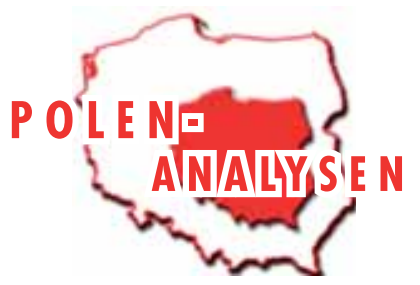

Wird der Beitritt Polens zur Europäischen Union die Zahl der Armen in Polen vergrößern oder verringern?

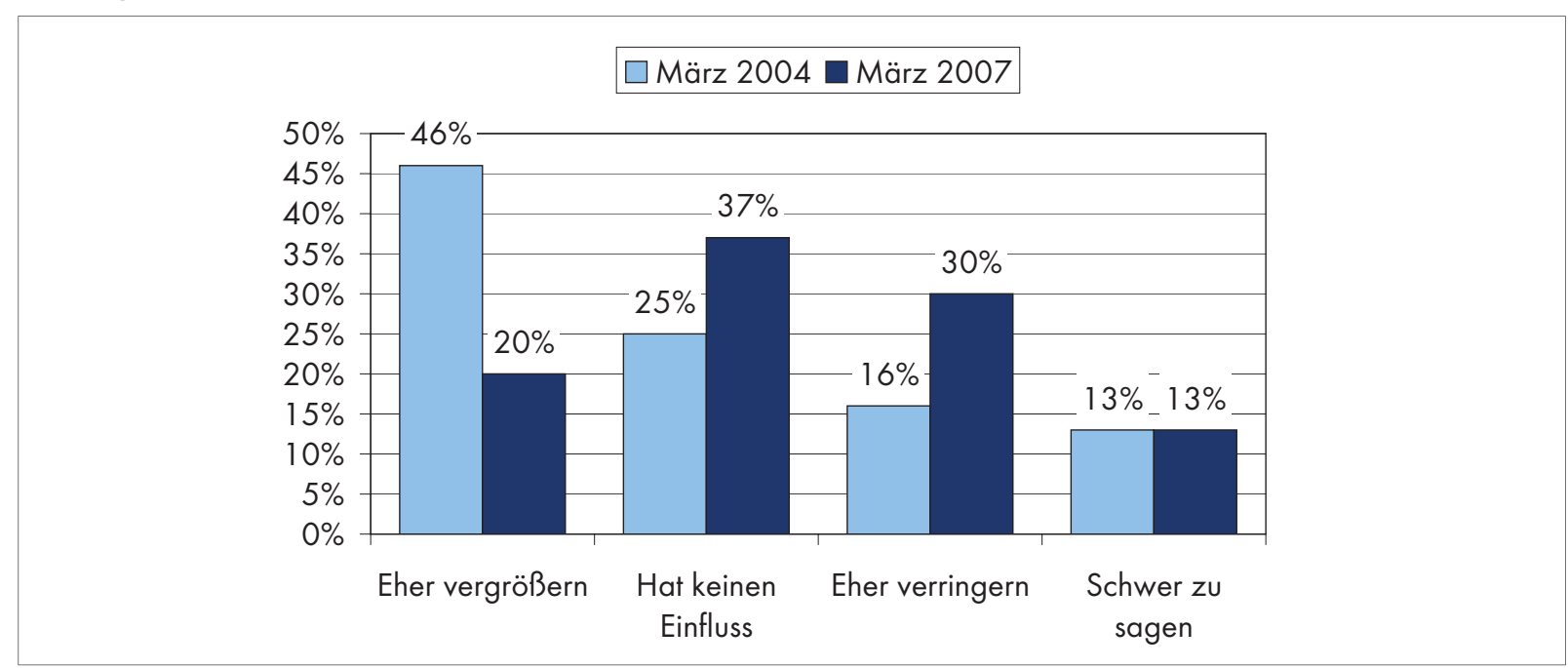

Quelle: CBOS BS/71/2007: Opinie na temat zasiegu biedy w Polsce [Meinungen zum Ausmaß der Armut in Polen], Warszawa, 04/2007. www.cbos.pl

Wie wird sich Ihrer Meinung nach im Laufe der nächsten Jahre die Zahl der Armen in Polen entwickeln? Es wird geben:

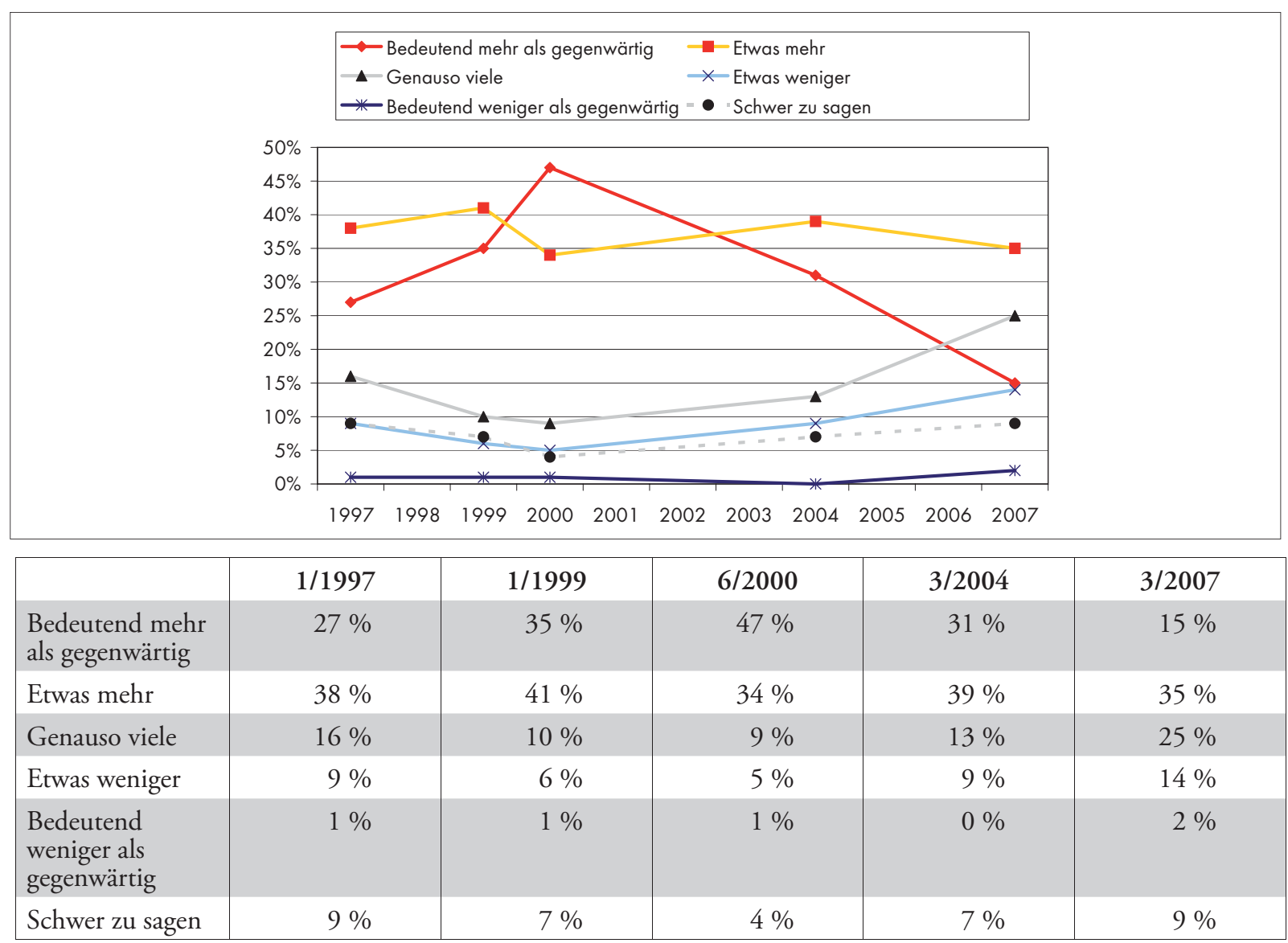

Quelle: CBOS BS/71/2007: Opinie na temat zasiegu biedy w Polsce [Meinungen zum Ausmaß der Armut in Polen], Warszawa, 04/2007. www.cbos.pl 


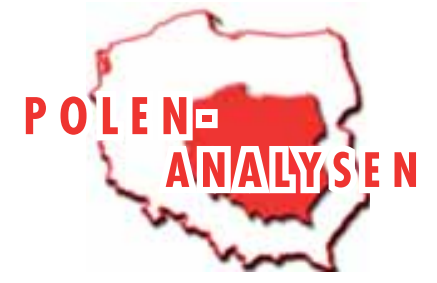

Was entscheidet Ihrer Meinung nach darüber, dass manche Menschen der Armut nicht entkommen?

\begin{tabular}{|c|c|c|c|c|c|}
\hline & $1 / 1997$ & $1 / 1999$ & $6 / 2000$ & $3 / 2004$ & $3 / 2007$ \\
\hline Arbeitslosigkeit & $58 \%$ & $67 \%$ & $79 \%$ & $79 \%$ & $53 \%$ \\
\hline Alkoholismus & $35 \%$ & $39 \%$ & $31 \%$ & $31 \%$ & $40 \%$ \\
\hline $\begin{array}{l}\text { fehlende Ausbildung / } \\
\text { berufl. Qualifikationen }\end{array}$ & $26 \%$ & $32 \%$ & $35 \%$ & $38 \%$ & $39 \%$ \\
\hline $\begin{array}{l}\text { Unbeholfenheit, } \\
\text { Hilflosigkeit bei der } \\
\text { Organisation des } \\
\text { eigenenLebens }\end{array}$ & $40 \%$ & $33 \%$ & $27 \%$ & $31 \%$ & $37 \%$ \\
\hline $\begin{array}{l}\text { Faulheit, keine Lust, eine } \\
\text { Arbeit auszuüben }\end{array}$ & $31 \%$ & $25 \%$ & $20 \%$ & $18 \%$ & $35 \%$ \\
\hline Krankheit, Behinderung & $41 \%$ & $37 \%$ & $27 \%$ & $23 \%$ & $27 \%$ \\
\hline $\begin{array}{l}\text { Fehlende staatliche } \\
\text { Unterstützung und } \\
\text { Fürsorge }\end{array}$ & $20 \%$ & $20 \%$ & $34 \%$ & $35 \%$ & $24 \%$ \\
\hline Niedrige Renten & $29 \%$ & $31 \%$ & $32 \%$ & $27 \%$ & $22 \%$ \\
\hline Schicksal & $5 \%$ & $5 \%$ & $5 \%$ & $5 \%$ & $5 \%$ \\
\hline $\begin{array}{l}\text { Fehlende familiäre } \\
\text { Unterstützung und } \\
\text { Fürsorge, keine Familie }\end{array}$ & $6 \%$ & $5 \%$ & $3 \%$ & $4 \%$ & $3 \%$ \\
\hline
\end{tabular}

Die Summe ergibt nicht $100 \%$, da drei Antworten gegeben werden konnten.

Quelle: CBOS BS/71/2007: Opinie na temat zasiegu biedy w Polsce [Meinungen zum Ausmaß der Armut in Polen], Warszawa, 04/2007. ww w.cbos.pl

Welche der Aussagen trifft auf Ihre gegenwärtige finanzielle Situation am meisten zu?

\begin{tabular}{|l|c|c|c|c|c|c|c|}
\hline $\begin{array}{l}\text { Ich bin zuversichtlich, } \\
\text { dass wir finanziell } \\
\text { zurechtkommen werden. }\end{array}$ & $11 \%$ & $15 \%$ & $11 \%$ & $11 \%$ & $11 \%$ & $11 \%$ & $21 \%$ \\
\hline $\begin{array}{l}\text { Ich habe keine Angst } \\
\text { vor Armut, befürchte } \\
\text { aber, dass sich unsere } \\
\text { materielle Situation } \\
\text { verschlechtern könnte. }\end{array}$ & $39 \%$ & $38 \%$ & $34 \%$ & $33 \%$ & $36 \%$ & $36 \%$ & $33 \%$ \\
$\begin{array}{l}\text { Ich habe Angst vor } \\
\text { Armut, denke aber, } \\
\text { dass wir irgendwie } \\
\text { zurechtkommen werden. }\end{array}$ & $37 \%$ & $33 \%$ & $38 \%$ & $37 \%$ & $36 \%$ & $37 \%$ & $35 \%$ \\
$\begin{array}{l}\text { Ich habe Angst vor } \\
\text { Armut und weiß nicht, } \\
\text { wie wir zurechtkommen } \\
\text { werden. }\end{array}$ & $12 \%$ & $13 \%$ & $16 \%$ & $18 \%$ & $15 \%$ & $15 \%$ & $9 \%$ \\
\hline \begin{tabular}{l} 
Schwer zu sagen \\
\hline
\end{tabular} & $1 \%$ & $1 \%$ & $1 \%$ & $1 \%$ & $2 \%$ & $1 \%$ & $2 \%$ \\
\hline
\end{tabular}

Quelle: CBOS BS/71/2007: Opinie na temat zasięgu biedy w Polsce [Meinungen zum Ausmaß der Armut in Polen], Warszawa, 04/2007. www.cbos.pl 


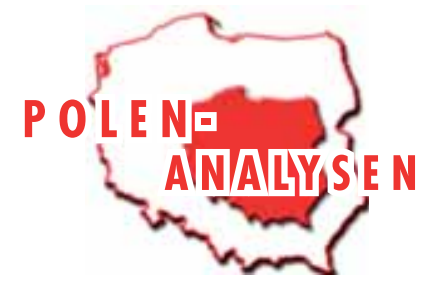

Sind Ihre Einkünfte ausreichend, um die Grundbedürfnisse (Wohnung, Ernährung, Kleidung usw.) zu befriedigen?

\begin{tabular}{|c|c|c|c|}
\hline & $3 / 2004$ & $3 / 2005$ & $3 / 2006$ \\
\hline Nicht ausreichend & $35 \%$ & $33 \%$ & $33 \%$ \\
\hline Fast ausreichend & $42 \%$ & $45 \%$ & $42 \%$ \\
\hline Vollkommen ausreichend & $22 \%$ & $20 \%$ & $24 \%$ \\
\hline Mehr als ausreichend & $1 \%$ & $2 \%$ & $1 \%$ \\
\hline
\end{tabular}

Quelle: CBOS, BS/54/2006: Oceny sytuacji materialnej [Beurteilungen der materiellen Situation], Warszawa, 03/2006, w w w.cbos.pl

Trat in den letzten zwölf Monaten die Situation ein, dass Ihnen Geld fehlte für:

\begin{tabular}{|l|c|c|c|c|c|c|c|c|}
\hline & $11 / 1995$ & $4 / 1996$ & $10 / 1996$ & $10 / 1997$ & $4 / 2002$ & $4 / 2003$ & $3 / 2005$ & $3 / 2006$ \\
\hline Urlaub & - & - & - & - & $60 \%$ & $59 \%$ & $57 \%$ & $59 \%$ \\
\hline $\begin{array}{l}\text { Einrichtungsgegenstände } \\
\text { und Geräte für den Haushalt }\end{array}$ & - & - & - & - & $58 \%$ & $60 \%$ & $57 \%$ & $57 \%$ \\
$\begin{array}{l}\text { Kulturelle Aktivitäten (Kauf } \\
\text { von Büchern, Theater-, }\end{array}$ & $47 \%$ & $48 \%$ & $43 \%$ & $42 \%$ & $41 \%$ & $44 \%$ & $42 \%$ & $44 \%$ \\
Konzertkarten etc.) & & & & & & & & \\
Kleidung, Schuhe & $57 \%$ & $56 \%$ & $47 \%$ & $46 \%$ & $38 \%$ & $39 \%$ & $38 \%$ & $36 \%$ \\
Arznei- und Heilmittel & $37 \%$ & $39 \%$ & $39 \%$ & $35 \%$ & $36 \%$ & $39 \%$ & $37 \%$ & $36 \%$ \\
\hline $\begin{array}{l}\text { Lebensmittel } \\
\text { Feste Kosten (Miete etc.) }\end{array}$ & - & - & - & - & - & $32 \%$ & $29 \%$ & $24 \%$ \\
\hline (Weiter-)Bildung & $22 \%$ & $20 \%$ & $21 \%$ & $22 \%$ & $20 \%$ & $23 \%$ & $22 \%$ & $21 \%$ \\
\hline Hygiene & $22 \%$ & $20 \%$ & $20 \%$ & $15 \%$ & $19 \%$ & $22 \%$ & $19 \%$ & $20 \%$ \\
\hline
\end{tabular}

Quelle: CBOS BS/54/2006: Oceny sytuacji materialnej [Bewertungen der materiellen Situation], Warszawa, 03/2006. w w w.cbos.p I 


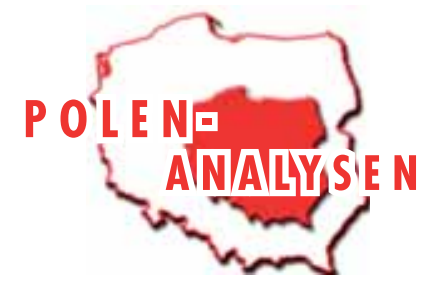

\section{Chronik}

\section{Vom 19. Februar bis zum 3. März 2008}

\begin{tabular}{|c|c|}
\hline 19.02.2008 & $\begin{array}{l}\text { Die Regierung nimmt den Gesetzesentwurf zur Ratifizierung des Vertrags von Lissabon an. Die Ratifizierung bedarf } \\
\text { der Zweidrittel-Mehrheit des Sejm und des Senats und wird formal vom Staatspräsidenten bestätigt. }\end{array}$ \\
\hline 20.02.2008 & $\begin{array}{l}\text { In einem Interview der Tageszeitung "Gazeta Wyborcza« spricht sich Ministerpräsident Donald Tusk für eine } \\
\text { Verfassungsdebatte zur Machtverteilung zwischen Ministerpräsident und Staatspräsident aus. }\end{array}$ \\
\hline 22.02.2008 & $\begin{array}{l}\text { Nach einem Bericht der Europäischen Kommission fiel die Arbeitslosigkeit in Polen im vergangenen Jahr von 11,8\% } \\
\text { auf 8,1 \% - der stärkste Rückgang unter den Ländern der Europäischen Union. Die Beschäftigungsquote liegt bei } \\
\text { Frauen in Polen unter } 50 \% \text { (EU-Ziel: Beschäftigung von } 60 \% \text { bis } 2010 \text { ) und bei älteren Arbeitnehmern unter } \\
30 \% \text { (EU-Ziel: } 50 \% \text { ). Die Gesamtbeschäftigungsquote beträgt ca. } 55 \% \text { und ist die niedrigste in der EU. Auf- } \\
\text { grund unterschiedlicher statistischer Methoden unterscheiden sich die EU-Daten von polnischen Statistiken. }\end{array}$ \\
\hline 23.02.2008 & $\begin{array}{l}\text { Der ehemalige Ministerpräsident und derzeitige Vertreter Polens bei der Europäischen Bank für Wiederaufbau und } \\
\text { Entwicklung (EBWE), Kazimierz Marcinkiewicz, bewertet die ersten } 100 \text { Tage der Regierung Tusk als "guten, in } \\
\text { einigen Bereichen sehr guten« Anfang. Der polnisch-polnische Krieg sei beendet worden, in der Außenpolitik seien } \\
\text { die Prioritäten beibehalten, aber der Stil positiv verändert worden, was sich auch auf ausländische Investitionen } \\
\text { in Polen positiv auswirke. }\end{array}$ \\
\hline 24.02.2008 & $\begin{array}{l}\text { Ministerpräsident Donald Tusk zieht in einer Regierungserklärung eine Bilanz der ersten } 100 \text { Tage seiner Amtszeit. } \\
\text { Bekräftigt werden u.a. Vorschläge zu einer Verfassungsänderung hinsichtlich einer Begrenzung der Immunität von } \\
\text { straffällig gewordenen Abgeordneten, zur Abschaffung der zweiten Kammer, des Senats, und einer Festschreibung } \\
\text { der Kompetenzen des Staatspräsidenten und des Ministerpräsidenten in der Verfassung sowie zur Streichung der } \\
\text { staatlichen Parteienfinanzierung und zur Einführung einer linearen Steuer. Darüber hinaus zeigt sich Tusk opti- } \\
\text { mistisch, was die wirtschaftliche Situation des Landes angeht. Polen könne bis } 2015 \text { zu den bestentwickelten } \\
\text { Ländern in der Europäischen Union aufschließen. }\end{array}$ \\
\hline 25.02.2008 & $\begin{array}{l}\text { Aus einem Bericht der Europäischen Kommission geht hervor, dass } 26 \% \text { der polnischen Kinder in Armut leben. } \\
\text { Polen ist damit zusammen mit Lettland Schlusslicht in der Europäischen Union. }\end{array}$ \\
\hline 26.02.2008 & $\begin{array}{l}\text { Der Ministerrat beschließt die Anerkennung der Unabhängigkeit des Kosovo. Staatspräsident Lech Kaczyński } \\
\text { hatte seine Vorbehalte mitgeteilt, er respektiere aber die Entscheidung der Regierung. }\end{array}$ \\
\hline 28.02.2008 & $\begin{array}{l}\text { Der Sejm beschließt, dass der EU-Reformvertrag vom Parlament ratifiziert werden wird. Ministerpräsident Donald } \\
\text { Tusk zeigt sich mit diesem Verfahrensweg zufrieden, da ein Referendum als Alternative an einer zu geringen } \\
\text { Bürgerbeteiligung scheitern könnte. }\end{array}$ \\
\hline 29.02.2008 & $\begin{array}{l}\text { Die Tageszeitung "Gazeta Wyborcza« meldet, dass die Präsidialkanzlei den polnischen Juden, die } 1968 \text { im Zuge } \\
\text { der staatlichen antisemitischen März-Kamapgne emigriert waren, als Geste der Wiedergutmachung die polnische } \\
\text { Staatsbürgerschaft bestätigen möchte. Derzeit wird auf die Antwort der Woiwoden gewartet, die allein die Staats- } \\
\text { bürgerschaft als nicht aberkannt bestätigen dürfen. Dabei herrscht Uneinigkeit, ob die Betroffenen nicht ihre } \\
\text { Staatsbürgerschaft verloren haben und folglich beim polnischen Staatspräsidenten eine neue beantragen müssten. } \\
\text { Inoffiziell wird die Befürchtung geäußert, dass eine Bestätigung der polnischen Staatsbürgerschaft auch von den } \\
\text { Bürgern, die in den 1960er-1980er Jahren zu Zehntausenden in die Bundesrepublik Deutschland emigrierten, in } \\
\text { Anspruch genommen werden kann, was Konsequenzen z.B. für Rückforderungen von Immobilien hätte. }\end{array}$ \\
\hline 01.03 .2008 & $\begin{array}{l}\text { Auf der Tagung des Landesrates der Bürgerplattform (Platforma Obywatelska-PO) werden u.a. Gesetzesvorhaben } \\
\text { zur Abschaffung der sog. Belka-Steuer - der Besteuerung von Sparguthaben - sowie der Rundfunk- und Fernseh- } \\
\text { gebühr für Rentner thematisiert. Außerdem soll bis März } 2009 \text { eine parteiinterne personelle und strukturelle } \\
\text { Evaluierung sowie eine Bestandsaufnahme des Parteivermögens vorgenommen werden. Außenminister Radosław } \\
\text { Sikorski und der stellvertretende Fraktionsvorsitzende Jarosław Gowin werden in den Vorstand des Landesrates } \\
\text { gewählt. }\end{array}$ \\
\hline 03.03 .2008 & Nach Einschätzung des Finanzministeriums lag die jährliche Inflationsrate im Februar bei 4,6 \%. \\
\hline
\end{tabular}




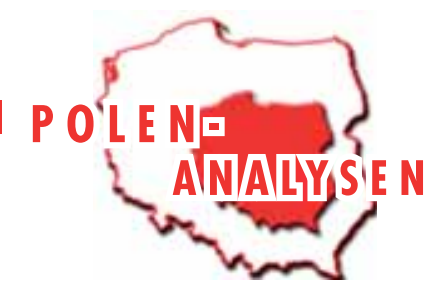

Über die Polen-Analysen

Die Polen-Analysen erscheinen zweimal monatlich als E-Mail-Dienst. Sie werden gemeinsam vom Deutschen PolenInstitut Darmstadt, der Bremer Forschungsstelle Osteuropa und der Deutschen Gesellschaft für Osteuropakunde herausgegeben. Die Herausgeber danken der BSH Bosch und Siemens Hausgeräte GmbH München und dem Stifterverband für die Deutsche Wissenschaft für ihre Unterstützung.

Ein Archiv der Polen-Analysen finden Sie im Internet unter w w w.laender-analysen.de/ polen

Für ein kostenloses Abonnement senden Sie eine E-Mail mit dem Stichwort PolenAnalysen in der Betreffzeile an polen-analysen@dpi-da.de

\title{
Deutsches Polen-Institut Darmstadt
}

Das Deutsche Polen-Institut Darmstadt (DPI) ist ein Forschungs-, Informations-, und Veranstaltungszentrum für polnische Kultur, Geschichte, Politik, Gesellschaft und die deutsch-polnischen Beziehungen, die sich im Kontext der europäischen Integration entwickeln. Das seit März 1980 aktive und bis 1997 von Gründungsdirektor Karl Dedecius geleitete Institut ist eine Gemeinschaftsgründung der Stadt Darmstadt, der Länder Hessen und Rheinland-Pfalz sowie des Bundes. Seit 1987 ist die Trägerschaft auf die Kultusministerkonferenz der Länder ausgedehnt. Einen wesentlichen Beitrag zur Verwirklichung der Institutsziele leisten private Stiftungen. Das DPI hat satzungsgemäß die Aufgabe, durch seine Arbeit zur Vertiefung der gegenseitigen Kenntnisse des kulturellen, geistigen und gesellschaftlichen Lebens von Polen und Deutschen beizutragen.

Ziel der Vermittlertätigkeit des DPI ist es, "die zu interessieren, auf die es politisch, wirtschaftlich, gesellschaftlich und kulturell im deutsch-polnischen Verhältnis ankommt (Leitlinien 1997). Es geht um die Entscheider und Multiplikatoren in Politik, Kultur, Bildung, Verwaltung, Medien und Wirtschaft und, wesentlich stärker ausgeprägt als bisher, um das Hineinwirken in Wissenschaft, Forschung und Bildung.

Derzeit bemüht sich das DPI in Kooperation mit den verstreuten Orten wissenschaftlicher Polen-Kompetenz an deutschen Hochschulen und Forschungsinstituten verstärkt darum, ausgehend von einer Bestandsaufnahme deutscher Polen-Forschung Ort wissenschaftlicher Forschung und verbindendes, vernetzendes und kooperierendes Zentrum zu werden. Ausgangspunkt der Neuausrichtung ist die kaum mehr kontrollierbare Dynamik des Rückbaus der Ressourcen der wissenschaftlichen Polen-Kompetenz in den unterschiedlichen Disziplinen. Mit der über 50.000 Bände zählenden multidisziplinären Fachbibliothek für Polen mit einer einzigartigen Sammlung polnischer Literatur in der Originalsprache und in deutscher Übersetzung ist das DPI bereits ein geschätzter Ort der Recherche und des wissenschaftlichen Arbeitens. (w w w.deutsches-polen-institutde)

\section{Forschungsstelle Osteuropa an der Universität Bremen}

1982 gegründet, widmet sich die Forschungsstelle Osteuropa an der Universität Bremen unter der Leitung von Prof. Dr. Eichwede kulturellen und gesellschaftlichen Entwicklungen der Länder Ost- und Ostmitteleuropas in Zeitgeschichte und Gegenwart. Die Forschungsstelle besitzt in ihrem Archiv eine einzigartige Sammlung alternativer Kulturgüter und unabhängiger Texte aus den ehemaligen sozialistischen Ländern. Darunter befindet sich auch eine umfangreiche Sammlung des "Zweiten Umlaufs«, die das Schrifttum und Dokumente unabhängiger Initiativen und gesellschaftlicher Gruppen in Polen aus der Zeit von 1976 bis zum Umbruch umfasst. Neben ausführlicher individueller Forschung zu Dissens und Gesellschaft im Sozialismus, leitet die Forschungsstelle seit Januar 2007 ein gemeinsames Projekt mit einem Verbund von internationalen Forschungsinstituten zum Thema »Das andere Osteuropa - die 1960 er bis 1980er Jahre, Dissens in Politik und Gesellschaft, Alternativen in der Kultur. Beiträge zu einer vergleichenden Zeitgeschichte«, welches von der VolkswagenStiftung finanziert wird.

Im Bereich der post-sozialistischen Gesellschaften sind in den letzten Jahren umfangreiche Forschungsprojekte durchgeführt worden, deren Schwerpunkte auf politischen Entscheidungsprozessen, Wirtschaftskultur und der EU-Osterweiterung lagen. Eine der Hauptaufgaben der Forschungsstelle ist die Information der interessierten Öffentlichkeit. Dazu gehören unter anderem regelmäßige E-Mail-Informationsdienste mit fast 15.000 Abonnenten in Politik, Wirtschaft und den Medien.

Mit ihrer in Deutschland einzigartigen Sammlung von Publikationen zu Osteuropa ist die Forschungsstelle eine Anlaufstelle sowohl für Wissenschaftler als auch für die interessierte Öffentlichkeit. In der Bibliothek sind derzeit neben anderen breit angelegten Beständen allein aus Polen ca. 300 laufende Periodika zugänglich. Die Bestände werden in Datenbanken systematisch erfasst. (w w w.fo rschung sstelle.uni-b remen.de)

\author{
Die Meinungen, die in den Polen-Analysen geäußert werden, geben ausschließlich die Auffassung der Autoren wieder. \\ Abdruck und sonstige publizistische Nutzung sind nach Rücksprache mit der Redaktion gestattet. \\ Redaktion: Prof. Dr. Dieter Bingen (Darmstadt), Dr. Stefan Garsztecki (Bremen), Silke Plate, M.A. (Bremen) \\ Technische Gestaltung: Matthias Neumann \\ Polen-Analysen-Layout: Cengiz Kibaroglu, Matthias Neumann \\ ISSN 1863-9712 (C) 2008 by Deutsches Polen-Institut Darmstadt und Forschungsstelle Osteuropa, Bremen \\ Kontakt: Dr. Andrzej Kaluza, Presse- und Öffentlichkeitsarbeit, Deutsches Polen-Institut, Mathildenhöhweg 2, \\ D-64287 Darmstadt, Tel.: 06151/4985-13, Fax: 06151/4985-10, E-Mail: polen-analysen@dpi-da.de, Internet: www.laender-analysen.de/polen
}

